\title{
Findings About Events or Interventions Numeric Result in Standard Unit
}

National Cancer Institute

\section{Source}

National Cancer Institute. Findings About Events or Interventions Numeric Result in

Standard Unit. NCI Thesaurus. Code C113369.

The numerical identifier of findings about events or interventions result in standard units. 